DOI: 10.1002/aenm.201100719

Submitted to

ENERGY

Article type: Full Paper

\title{
Building on Soft Foundations - New Possibilities for Controlling Hybrid Photovoltaic Architectures
}

Joseph B. Franklin, Jonathan M. Downing, Finn Giuliani, Mary P. Ryan and Martyn A. McLachlan*

J. B. Franklin ${ }^{1}$, J. M. Downing ${ }^{1}$, F. Giuliani ${ }^{2}$, M. P. Ryan ${ }^{3}$ and M. A. McLachlan ${ }^{1}$

${ }^{1}$ Department of Materials and Centre for Plastic Electronics, Imperial College London, London SW7 2AZ, United Kingdom

${ }^{2}$ Department of Materials and Department of Mechanical Engineering, Imperial College London, London SW7 2AZ, United Kingdom

${ }^{3}$ Department of Materials and London Centre for Nanotechnology, Imperial College

London, London SW7 2AZ, United Kingdom

E-mail: martyn.mclachlan@imperial.ac.uk

Keywords: Zinc Oxide, Conjugated Polymers, Organic Electronics, Thin Films, Hybrid Materials

\begin{abstract}
Here we outline a methodology for the deposition of a highly crystalline transparent conductive metal oxide $(\mathrm{ZnO})$ onto a functionalised organic thin film poly (3-hexylthiophene, P3HT) without degradation of the microstructural, optical or electronic properties of the organic layer. To confirm the absence of damage we have assembled a simple bilayer photovoltaic (PV) device. The processing methodology has enabled us to demonstrate hybrid photovoltaic (h-PV) device formation in the conventional architecture for the first time. The compatibility of this novel low temperature processing route with $\pi$-conjugated molecular materials has tremendous potential for applications including electron accepting layers and optical spacers in organic PVs and light emitting diodes, as transparent electrodes and all future devices reliant on flexible substrates.
\end{abstract}




\section{Introduction}

Submitted to MATERIALS

Hybrid photovoltaic (h-PV) devices combine the favourable processing and absorption characteristics of $\pi$-conjugated molecular materials with the stability and electrical properties of inorganic materials. ${ }^{[1]}$ In particular, the combination of a wide band-gap metal oxide e.g. $\mathrm{ZnO}$ or $\mathrm{TiO}_{2}$ with a conjugated polymer presents a pairing of materials suitable for the production of scalable, stable, nanostructured and ultimately more efficient photovoltaic devices. Despite this promise, h-PVs have yet to be prepared with efficiencies approaching even modest organic photovoltaics (OPVs). Attempts to address this challenge have mostly focused on morphological and microsturctural control of the active layer. ${ }^{[2-4]}$ At present, h-PV devices are prepared either by $i$ ) deposition of the organic phase into a pre-grown metal-oxide layer, ${ }^{[5,6]}$ or ii) co-deposition of both the inorganic and organic species. ${ }^{[4]}$ The deposition of highly crystalline metal-oxide directly on to any $\pi$-conjugated functional material, whilst maintaining the inherent integrity and properties of the organic layer, has yet to be demonstrated - primarily due to the elevated deposition or annealing temperatures of vacuum based processes or the requirements for substrate conductivity or harsh chemical conditions for solution-based processing methods.

For PV devices two distinct architectures may be prepared, namely the conventional ${ }^{[17]}$ and the so-called inverted ${ }^{[18]}$ structures. The conventional geometry requires first the deposition of the organic component onto the transparent electrode, typically indium tin oxide (ITO) coated glass, followed by the deposition of the organic material and metallic electrode. The preparation of the conventional configuration is more desirable as the transparent and metallic electrodes act as hole and electron acceptors respectively. Additionally, there is no contact between the metallic electrode and the organic material - thus avoiding unwanted reactions at this interface which have been shown to have a significant contribution to cell degradation. ${ }^{[19,}$ 
20]. However the processing conditions used currently for oxide deposition mean that the inverted architecture has been the only reported h-PV structure thus far.

The necessity for developing techniques that allow metal oxides to be deposited onto functional organic materials extend far beyond h-PVs. For example, in the development of transparent conductive oxides (TCOs) on flexible substrates, optical spacers in solar cells ${ }^{[7,8]}$ and LEDs, ${ }^{[9-11]}$ electron accepting layers in OPV devices ${ }^{[12,13]}$ and multilayer devices. ${ }^{[14]}$ Here we demonstrate the use of pulsed laser deposition (PLD) as a method for depositing highly crystalline $\mathrm{ZnO}$ at low temperatures $\left(200{ }^{\circ} \mathrm{C}\right)$ directly on to an functional organic thin film, poly(3-hexylthiophene) (P3HT). Importantly the oxide deposition conditions identified preserve the optical and electronic properties of the P3HT. This versatile and well-studied deposition technique allows precise control of the oxide film thickness, orientation and stoichiometry. ${ }^{[15]}$ Whilst PLD of indium tin oxide (ITO) has been previously demonstrated on flexible substrates ${ }^{[16]}$ we believe that this is the first demonstration of direct oxide deposition onto a functional organic material - and to the best of our knowledge the creation of the first conventional architecture h-PV device. The development of a reproducible methodology for preparing conventional architecture h-PV paves the pathway to effective studies into processing-performance relationships in h-PVs.

\section{Thin Film Characterization}

The X-ray diffraction (XRD) pattern of a $\mathrm{ZnO}$ film deposited on a $40 \mathrm{~nm}$ film of P3HT at $200^{\circ} \mathrm{C}$ (background of 50 mtorr $\mathrm{O}_{2}$ ) on an ITO coated glass substrate is shown in Figure 1a. A highly crystalline film with a preferential (002) orientation is deposited i.e. the c-axis of the unit cell is aligned perpendicular to the substrate. In addition to the $\mathrm{ZnO}(002)$ family of diffraction peaks, ITO substrate peaks are observed at lower intensity. The inset (figure 1b) shows in detail the region of the $\mathrm{ZnO}(002)$ diffraction peak measured from films deposited 
under different oxygen pressures. At 50 mtorr the measured full width at half-maximum (FWHM) of the (002) diffraction peak is $0.40^{\circ} 2 \theta$, the peak position of $34.53^{\circ} 2 \theta$ is close to that of a measured single crystal $34.55^{\circ} 2 \theta$. A reduction in background oxygen pressure $(<50$ mtorr) shifts the (002) diffraction peak to lower $2 \theta$ values, accompanied by an increase in the measured FWHM and a reduction in the peak area. In contrast, as the oxygen pressure is increased (> 50 mtorr) there is no systematic shift of the (002) diffraction peak or the measured FWHM however there is a reduction in the peak area.

\section{Figure 1}

Table 1

Background oxygen is necessary in PLD of $\mathrm{ZnO}$ to compensate for the zinc-rich species ejected from the target, ${ }^{[21]}$ additionally collisions with molecular oxygen in the chamber reduce the velocity of the ejected species arriving at the substrate. Therefore at low background oxygen pressure the ejected species will have minimal interaction with $\mathrm{O}_{2}$ and will arrive at the substrate with a large amount of kinetic energy, which facilitates reorganisation at the substrate resulting in improved crystallinity. ${ }^{[15]}$ The observed shift in the (002) diffraction peak position is attributed to the formation of oxygen deficient films, at $\sim 50$ mtorr $\mathrm{O}_{2}$ stoichiometry is achieved ${ }^{[15]}$ with no further shifts in peak position with increasing molecular oxygen content. The reduced peak intensity for films deposited at background oxygen pressures of 100 and 250 mtorr is attributed to two factors, firstly the reduced film thickness (Table 1) and secondly reduced crystallinity owing to interaction of the ejected species with molecular oxygen.

\section{Film Morphology}


Figures 2a-c show how $\mathrm{ZnO}$ film morphology changes with variation in oxygen pressure. The films deposited at $<50$ mtorr (Fig. 2a) typically show characteristic surface undulations which are attributed to the deformation of the underlying P3HT thin film, caused by the high energy incident particles. Increasing the oxygen pressure to $250 \mathrm{mtorr}$ results in macroscopic crack formation in the films (Fig. 2b). The defect formation energy for oxygen interstitials $\left(\mathrm{O}_{\mathrm{i}}\right)$ and zinc vacancies $\left(\mathrm{V}_{\mathrm{Zn}}\right)$ is reduced as oxygen pressure increases. ${ }^{[22]}$ Although here we do not characterize such defects we speculate that at the highest oxygen pressure investigated (250 mtorr) the film defect density is increased, causing accumulation of strain leading to cracking. The slight shift in the (002) diffraction peak at 250 mtorr to a lower $2 \theta$ value is also indicative of the presence of strain in the film. At a background oxygen pressure of $50 \mathrm{mtorr}$ a continuous, flat $\mathrm{ZnO}$ film is deposited across the entire substrate $(12 \times 12 \mathrm{~mm})-$ the observed grain size of around $30 \mathrm{~nm}$ (Fig. 2c) is consistent with XRD measurements (Table 1).

A transmission electron microscope (TEM) cross-section of a multilayer device containing a $\mathrm{ZnO}$ layer deposited at 50 mtorr is shown in Figure 2d. Each layer in the device can be clearly observed and is labelled for clarity. Importantly the interface between the P3HT and the $\mathrm{ZnO}$ is extremely sharp and no $\mathrm{ZnO}$ is observed to have damaged or penetrated the underlying P3HT layer (Fig. 2e). The columnar morphology of $\mathrm{ZnO}$ film is clearly visible, which is anticipated owing to the preferential (002) orientation ${ }^{[23]}$

\section{Figure 2}

\section{Optical Measurements and PV Device Characteristics}

Figure 3a shows the optical transmission spectra of a pristine $\mathrm{P} 3 \mathrm{HT}$ film and $\mathrm{P} 3 \mathrm{HT} / \mathrm{ZnO}$ multilayer films prepared as conventional and inverted device architectures. The sharp $\mathrm{ZnO}$ absorption at around $370 \mathrm{~nm}(\sim 3.25 \mathrm{eV})$, is observed in all $\mathrm{ZnO}$ films prepared at all oxygen pressures. The broader absorption bands between 450 and $650 \mathrm{~nm}$ are attributed to $\pi-\pi^{*}$ 
Submitted to MATERIALS

transitions in the P3HT, the small shoulder at around $625 \mathrm{~nm}$ is indicative of inter and intra chain ordering. ${ }^{[24]}$ These are observed on all of the structures prepared and with no absorption quenching $^{[25]}$ confirming our structural observation that the PLD of $\mathrm{ZnO}$ does not damage the P3HT. The UV-Vis data and TEM images confirm that no microstructural damage has occurred to the P3HT during the $\mathrm{ZnO}$ deposition.

\section{Figure 3}

The current-voltage $(\mathrm{J}-\mathrm{V})$ characteristics under dark and illumination of a conventional architecture device (ITO/40nm P3HT/150nm ZnO, $200{ }^{\circ} \mathrm{C}$ (50 mtorr)) are shown in figure $3 \mathrm{~b}$. The measured short circuit current $\left(\mathrm{J}_{\mathrm{sc}}\right)$, open circuit voltage $\left(\mathrm{V}_{\mathrm{oc}}\right)$, and fill factor were $0.18 \mathrm{mAcm}^{-2}, 0.18 \mathrm{~V}$, and 0.3 respectively. The calculated power conversion efficiency (PCE) was $0.01 \%$.' this modest performance is comparable to inverse $\mathrm{ITO} / \mathrm{ZnO} / \mathrm{P} 3 \mathrm{HT}$ devices reported previously. ${ }^{[5,26]}$ Here the remarkably flat interface between the P3HT and ZnO (Fig 2d-e), is likely to contribute significantly to the low PCE by minimising the interfacial area between polymer and oxide". Device improvements could be achieved through interfacial roughening, optimization of layer thickness, electrode choice and processing conditions. However, the preparation of a conventional architecture h-PV device for the first time represents a significant and necessary step in h-PV development, and the methodology is applicable for a wider range of optoelectronic devices.

\section{Conclusions}

We have demonstrated for the first time the deposition of highly crystalline $\mathrm{ZnO}$ onto an organic functionalized substrate (P3HT) using pulsed laser deposition (PLD). By tuning the background oxygen pressure in the PLD chamber a deposition regime has been identified in which stoichiometric $\mathrm{ZnO}$ can be deposited without degradation or damage to the P3HT. At

\footnotetext{
* Planar ITO/P3HT/Ag and ITO/ZnO/Ag structures were prepared and their J-V measured under dark and AM 1.5 illumination. In both cases no photocurrent was detected with only diode behavior observed.
} 
Submitted to MATERIALS low oxygen pressure, ( 5 mtorr), the $\mathrm{ZnO}$ deposited is oxygen deficient and physical damage to underlying $\mathrm{P} 3 \mathrm{HT}$ is observed owing to the high particle energy impacting on the soft $\mathrm{P} 3 \mathrm{HT}$. At high oxygen pressure ( $>100$ mtorr) the deposited films show extended cracks and surface defects. At 50 mtorr flat, continuous and stoichiometric $\mathrm{ZnO}$ layers can be deposited with no induced changes in the microstructure, optical or electronic properties of the $\mathrm{P} 3 \mathrm{HT}$. We have shown, for the first time that conventional architecture h-PV devices can be successfully prepared and predict improvements in device performance through modifications of the layer structure and cell configuration. The methodology presented represents an important step in comparing the device performance and photophysical processes in conventional and inverted architecture h-PV devices. More importantly, the wider requirement for transparent conducting oxides i.e. as optical spacers in multilayer devices, transparent top-electrodes various device technologies can be achieved using this methodology.

\section{Experimental Section}

P3HT deposition: Films were deposited on to ITO coated glass substrates (PsiOtec, UK Ltd,), cleaned by sequential washing in acetone, isopropanol and de-ionised water. Atomic force microscopy (AFM) and 4-point probe measurements of the bare substrates yields RMS roughness and sheet resistance values of $\sim 3.5 \mathrm{~nm}$ and $12-15 \Omega / \mathrm{sq}$ respectively. Precipitatefree P3HT films were spin-coated at $1500 \mathrm{rpm}$ for $30 \mathrm{~s}$ (Merck RR $120 \mathrm{~kg} \cdot \mathrm{mol}^{-1}, 15 \mathrm{mg} \cdot \mathrm{ml}^{-1}$ P3HT in chlorobenzene).

$\mathrm{ZnO}$ deposition: PLD apparatus and experimental methods are described in detail elsewhere. ${ }^{[15]}$ Briefly, a $\mathrm{KrF}$ laser with wavelength $248 \mathrm{~nm}$ was used with a pulse duration of 25 ns. A $\mathrm{ZnO}$ target measuring $22 \mathrm{~mm}$ diameter was prepared from $\mathrm{ZnO}$ powder (Aldrich $99.999 \%$ pressed and sintered at $1000{ }^{\circ} \mathrm{C}$ under flowing oxygen for 10 hours. Laser frequency, target-substrate distance and spot size were fixed for all experiments at $8 \mathrm{~Hz}, 50$ $\mathrm{mm}$ and $20 \mathrm{~mm}^{2}$ respectively. Substrates were held at $200{ }^{\circ} \mathrm{C}$ for 15 minutes prior to 


\section{Submitted to}

ENERGY

deposition. The laser power used was set at $500 \mathrm{~mJ}$ leading to a laser power density of 0.82 $\mathrm{J} . \mathrm{cm}^{-2}$, for all experiments the number of pulses was fixed at 5000. A background pressure of $3 \times 10^{-5}$ mtorr was maintained and the oxygen pressure was varied $(5,25,50,100,250$ mtorr). Following growth the films were allowed to cool to room temperature. No post-annealing steps were performed.

Electron microscopy: Film morphology and thicknesses were assessed using a LEO Gemini 1525 field emission gun scanning electron microscope (FEG-SEM), operating at 5-10 kV. Cross-sections were prepared using a FEI Helios dual-beam FIB-SEM and subsequent TEM images using a JEOL 2000 FX TEM.

X-ray diffraction measurements: XRD measurements were performed with a PANalytical $\mathrm{X}^{\prime}$ Pert pro MPD diffractometer equipped with an $\mathrm{X}^{\prime}$ 'celerator detector using $\mathrm{Cu} \mathrm{K} \alpha$ radiation $(\lambda=1.5418 \AA)$, operated at $40 \mathrm{kV}$ and $40 \mathrm{~mA}$ in conventional reflection mode.

UV-Vis absorption spectroscopy: UV-Vis spectroscopy was carried out using a monochromated white light source $(300-900 \mathrm{~nm})$ attached to a custom optical bench, controlled by BenWin+ software.

Device performance assessment: Three $150 \mathrm{~nm}$ thick silver contacts (1 x $8 \mathrm{~mm}$ each) were evaporated on the multilayer structures. Measurements were obtained using a Newport solar simulator with an AM 1.5 output, the current measured during dark and light scans over the range $(-0.5-0.5 \mathrm{~V})$ were obtained using a Keithley 2400 sourcemeter, all measurements were carried out in air.

\section{Acknowledgements}

The authors are grateful to Dr Peter Petrov (Imperial) and Professor David McComb (now at Ohio State University) for useful discussions and technical advice. J.B.F. and J.D. are grateful to the EPSRC for PhD studentships. M.A.M is funded by the Royal Academy of Engineering through a Research Fellowship. 


\section{References}

Submitted to MATERIAS

Received: ((will be filled in by the editorial staff))

Revised: ((will be filled in by the editorial staff))

Published online: ((will be filled in by the editorial staff))

[1] A. J. Said, G. Poize, C. Martini, D. Ferry, W. Marine, S. Giorgio, F. Fages, J. Hocq, J. Bouclé, J. Nelson, J. R. Durrant, J. Ackermann, The Journal of Physical Chemistry C 2010, 114, 11273.

[2] P. Atienzar, T. Ishwara, B. N. Illy, M. P. Ryan, B. C. O'Regan, J. R. Durrant, J. Nelson, The Journal of Physical Chemistry Letters 2010, 1, 708.

[3] Y.-J. Lee, M. T. Lloyd, D. C. Olson, R. K. Grubbs, P. Lu, R. J. Davis, J. A. Voigt, J. W. P. Hsu, The Journal of Physical Chemistry C 2009, 113, 15778.

[4] S. D. Oosterhout, M. M. Wienk, S. S. van Bavel, R. Thiedmann, L. Jan Anton Koster, J. Gilot, J. Loos, V. Schmidt, R. A. J. Janssen, Nature Materials 2009, 8, 818.

[5] D. C. Olson, Y.-J. Lee, M. S. White, N. Kopidakis, S. E. Shaheen, D. S. Ginley, J. A. Voigt, J. W. P. Hsu, The Journal of Physical Chemistry C 2008, 112, 9544.

[6] L. Baeten, B. Conings, H.-G. Boyen, J. D’Haen, A. Hardy, M. D’Olieslaeger, J. V. Manca, M. K. Van Bael, Advanced Materials 2011, doi: 10.1002/adma.201100414.

[7] J. Y. Kim, S. H. Kim, H. H. Lee, K. Lee, W. Ma, X. Gong, A. J. Heeger, Advanced Materials 2006, 18, 572.

[8] J. Gilot, I. Barbu, M. M. Wienk, R. A. J. Janssen, Applied Physics Letters 2007, 91, 113520.

[9] J.-A. Jeong, et al., Journal of Physics D: Applied Physics 2010, 43, 465403.

[10] M. Sessolo, H. J. Bolink, Advanced Materials 2011, 23, 1829.

[11] N. Bano, S. Zaman, A. Zainelabdin, S. Hussain, I. Hussain, O. Nur, M. Willander, Journal of Applied Physics 2010, 108, 043103.

[12] S. Schumann, R. Da Campo, B. Illy, A. C. Cruickshank, M. A. McLachlan, M. P. Ryan, D. J. Riley, D. W. McComb, T. S. Jones, Journal of Materials Chemistry 2011, 21, 2381.

[13] J. C. Wang, W. T. Weng, M. Y. Tsai, M. K. Lee, S. F. Horng, T. P. Perng, C. C. Kei, C. C. Yu, H. F. Meng, Journal of Materials Chemistry 2010, 20, 862.

[14] J. Gilot, M. M. Wienk, R. A. J. Janssen, Applied Physics Letters 2007, 90, 143512.

[15] J. B. Franklin, B. Zou, P. Petrov, D. W. McComb, M. P. Ryan, M. A. McLachlan, Journal of Materials Chemistry 2011, 21 (22), $8178-8182$.

[16] H. Kim, J. S. Horwitz, G. P. Kushto, Z. H. Kafafi, D. B. Chrisey, Applied Physics Letters 2001, 79, 284.

[17] S. E. Shaheen, C. J. Brabec, N. S. Sariciftci, F. Padinger, T. Fromherz, J. C. Hummelen, Applied Physics Letters 2001, 78, 841.

[18] Y. Sahin, S. Alem, R. de Bettignies, J.-M. Nunzi, Thin Solid Films 2005, 476, 340.

[19] M. T. Lloyd, D. C. Olson, P. Lu, E. Fang, D. L. Moore, M. S. White, M. O. Reese, D. S. Ginley, J. W. P. Hsu, Journal of Materials Chemistry 2009, 19, 7638.

[20] J. Weickert, R. B. Dunbar, H. C. Hesse, W. Wiedemann, L. Schmidt-Mende, Advanced Materials 2011, 23, 1810.

[21] F. Claeyssens, A. Cheesman, S. J. Henley, M. N. R. Ashfold, Journal of Applied Physics 2002, 92, 6886.

[22] K. Ellmer, A. Klein, B. Rech, Transparent Conductive Zinc Oxide Basics and Applications in Thin Film Solar Cells, Vol. 104, Springer, 2008. 


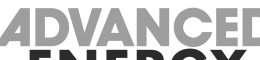 \\ Submitted to MATERIALS}

[23] T. Nobis, E. M. Kaidashev, A. Rahm, M. Lorenz, M. Grundmann, Physical Review Letters 2004, 93, 103903.

[24] C.-H. Wu, H. Li, H. H. Fong, V. A. Pozdin, L. A. Estroff, G. G. Malliaras, Organic Electronics 2011, 12, 1073.

[25] V. Shrotriya, J. Ouyang, R. J. Tseng, G. Li, Y. Yang, Chemical Physics Letters 2005, 411, 138.

[26] M. S. White, D. C. Olson, N. Kopidakis, A. M. Nardes, D. S. Ginley, J. J. Berry, Physica Status Solidi (a) 2010, 207, 1257. 
Submitted to MATERIALS

Figure 1 a) A typical XRD pattern for $\mathrm{ZnO}$ film deposited at $200{ }^{\circ} \mathrm{C}, 50$ mtorr, showing characteristic (002) $\mathrm{ZnO}$ peaks and substrate peaks, b) shows the observed change in peak position and intensity with oxygen pressure (5-250 mtorr).
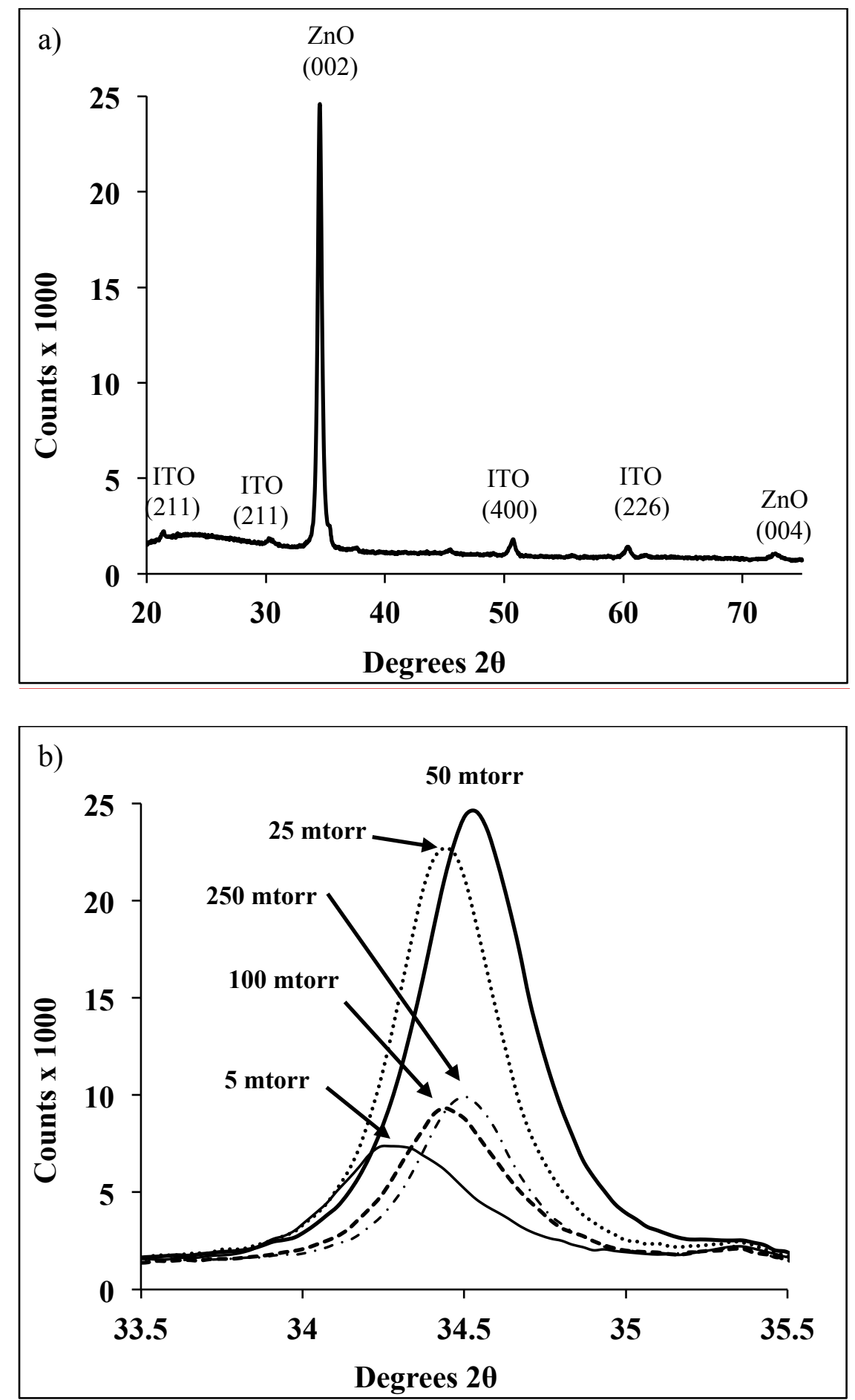


\section{ENERGY \\ Submitted to MATERIALS}

Figure 2 SEM micrographs showing surface the morphology of films deposited at a background oxygen pressure of; a) 5 mtorr, b) 250 mtorr, c) 50 mtorr. TEM micrographs showing d) individual layers of a multilayer conventional architecture h-PV device and e) detailed view of $\mathrm{P} 3 \mathrm{HT} / \mathrm{ZnO}$ interface showing no visible damage layer. Note that the Au and Pt layers were deposited for FIB imaging and sample preparation.

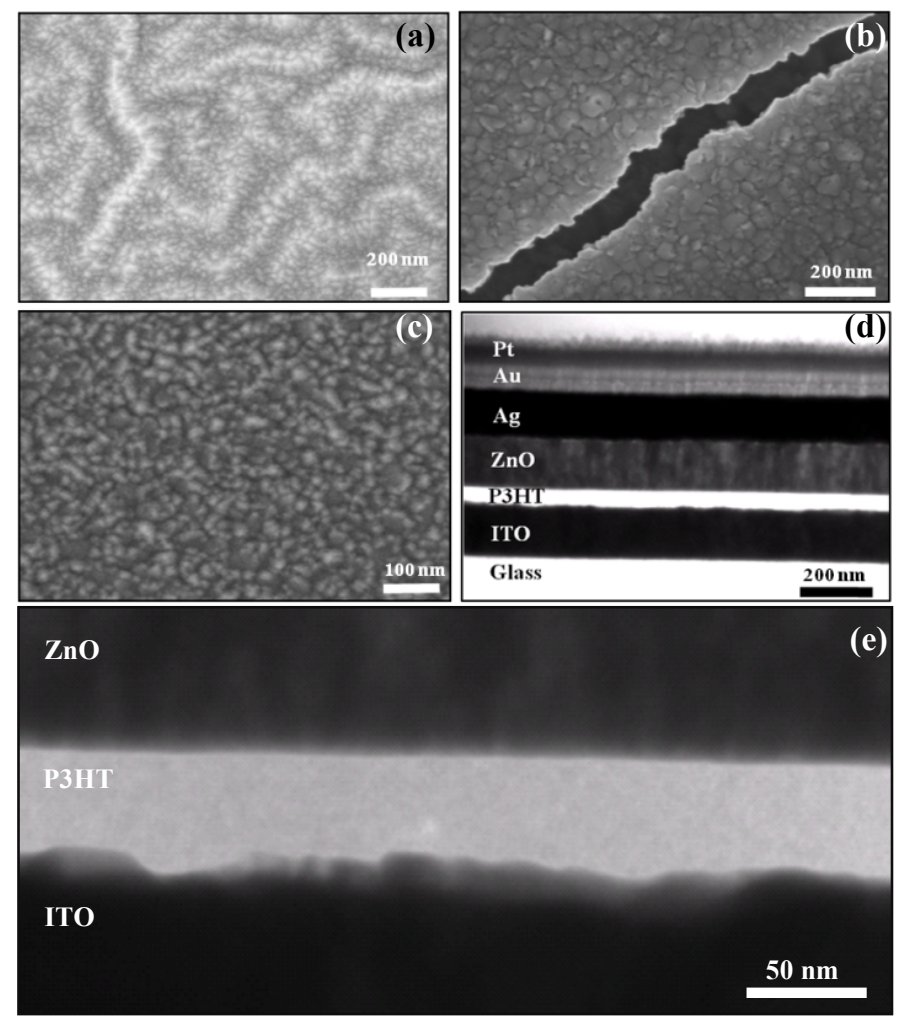


Submitted to MATERIALS

Figure 3 a) shows UV-vis transmission spectra for ITO/P3HT, ITO/P3HT/ZnO and $\mathrm{ITO} / \mathrm{ZnO} / \mathrm{P} 3 \mathrm{HT}$ films (ZnO deposited at 50 mtorr, $200{ }^{\circ} \mathrm{C}$ ), b) $\mathrm{J}-\mathrm{V}$ data for ITO/P3HT/ZnO/Ag device shown in figure 2d-e).
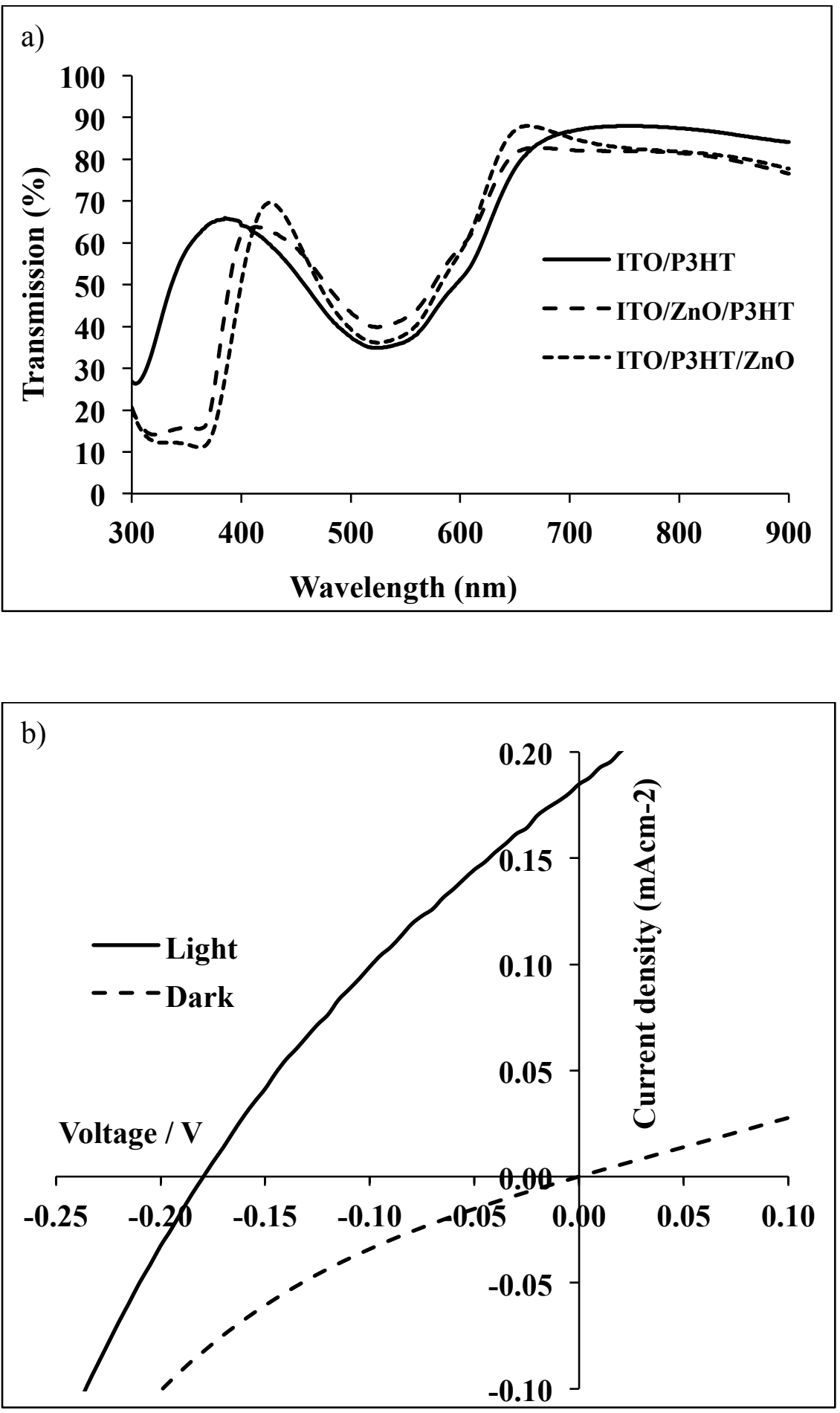
Submitted to MNERGY

Table 1 Peak position, full width at half-maximum (FWHM), thickness and grain size calculated by the Scherrer method of the $\mathrm{ZnO}$ film, extracted from the XRD data in Figure 1

\begin{tabular}{|c|c|c|c|c|}
\hline $\begin{array}{c}\text { Oxygen pressure } \\
\text { (mtorr) }\end{array}$ & $\begin{array}{c}\text { (002) peak } \\
\text { position }^{\circ} 2 \theta\end{array}$ & $\begin{array}{c}\text { FWHM } \\
{ }^{\circ} 2 \theta\end{array}$ & $\begin{array}{c}\text { Film thickness } \\
(\mathrm{nm})\end{array}$ & $\begin{array}{c}\text { Grain size } \\
(\mathrm{nm})\end{array}$ \\
\hline 5 & 34.30 & 0.47 & 160 & $213 \pm 3$ \\
\hline 25 & 34.44 & 0.37 & 166 & $287 \pm 5$ \\
\hline 50 & 34.53 & 0.40 & 170 & $260 \pm 4$ \\
\hline 100 & 34.57 & 0.38 & 124 & $277 \pm 5$ \\
\hline 250 & 34.50 & 0.35 & 128 & $308 \pm 6$ \\
\hline
\end{tabular}




\section{ToC entry}

The deposition of highly crystalline $\mathrm{ZnO}$ onto P3HT using pulsed laser deposition at low temperature is discussed. To demonstrate the applicability of the technique we outline for the first time the preparation of a conventional architecture hybrid photovoltaic device. No degradation of the microstructure, optical or electronic properties of the P3HT were observed. The methodology is widely applicable for depositing oxide interlayers and optical spacers in multilayer organic devices.

Keyword: Zinc Oxide, Conjugated Polymers, Organic Electronics, Thin Films, Hybrid Materials

Joseph B. Franklin, Jonathan Downing, Finn Giuliani, Mary P. Ryan and Martyn A. McLachlan*

Building on Soft Foundations - New Possibilities for Controlling Hybrid Photovoltaic Architectures

ToC figure

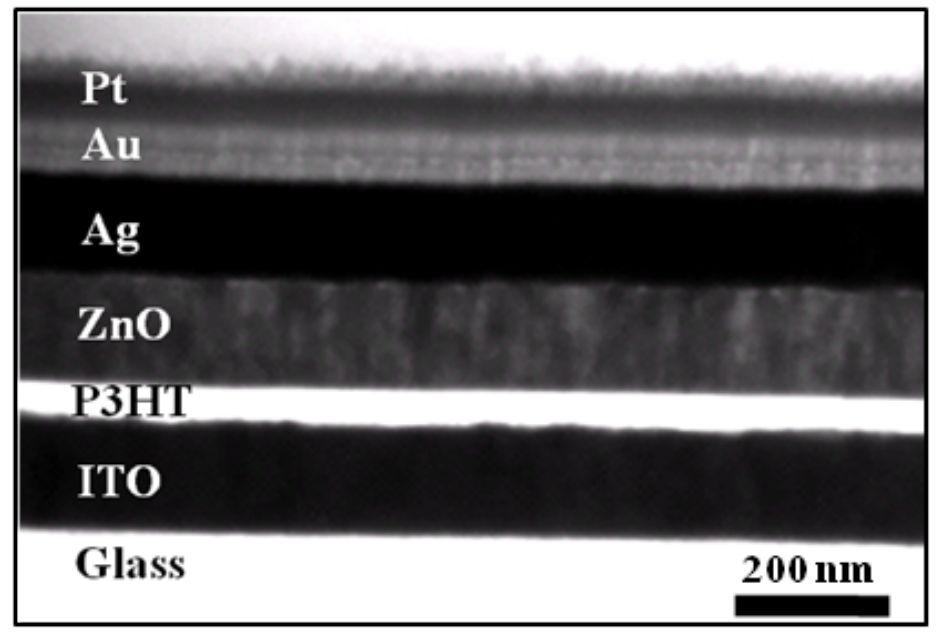

